\title{
ZFAT gene variant association with multiple sclerosis in the Arabian Gulf population: A genetic basis for gender-associated susceptibility
}

\author{
SONIA BOURGUIBA-HACHEMI ${ }^{1}$, TEBAH K. ASHKANANI ${ }^{1}$, FATEMA J. KADHEM ${ }^{1}$, \\ WASSIM Y. ALMAWI ${ }^{2}$, RAED ALROUGHANI ${ }^{3}$ and M. DAHMANI FATHALLAH ${ }^{1}$
}

${ }^{1}$ Department of Life Sciences, Biotechnology Program, College of Graduate Studies; ${ }^{2}$ Department of Medical Biochemistry, Arabian Gulf University, Manama 329, Bahrain; ${ }^{3}$ Division of Neurology, Department of Medicine,

Amiri Hospital, Kuwait City 13041, Kuwait

Received November 17, 2015; Accepted June 27, 2016

DOI: $10.3892 / \mathrm{mmr} .2016 .5692$

\begin{abstract}
Single nucleotide polymorphisms (SNPs) are useful genetic markers to investigate the onset of multiple sclerosis (MS). A genome wide association study identified 7 SNPs associated with interferon- $\beta$ therapy response, however, not with MS risk in a Spanish population. To investigate these findings in a different cohort, the 7 SNPs were investigated in an Arabian Gulf population. The SNPs were analyzed in 268 subjects (156 patients and 112 healthy volunteers) from the Arabian Gulf region using restriction fragment length polymorphism-polymerase chain reaction (PCR) and KBioscience Competitive Allele Specific PCR genotyping methods. Associations between the SNPs and MS were investigated using logistic regression. The present study observed, for the first time, that in an Arabian Gulf population, the ZFAT rs733254 polymorphism ( $\mathrm{T}>\mathrm{G}$ ) is a gender-specific risk marker for MS. ZFAT was associated with MS in women but not in men. The $\mathrm{G}$ variant was highly associated with the risk of MS [odds ratio $(\mathrm{OR})=2.38$ and $95 \%$ confidence interval $(\mathrm{CI})$, 1.45-3.91); $\mathrm{P}=0.0014]$. Whereas variant $\mathrm{T}$ was a significantly protective factor $[\mathrm{OR}=0.420(95 \% \mathrm{CI}, 0.25-0.69) ; \mathrm{P}=0.0014$, recessive model]. The findings of the present study provide a genetic basis for the gender-associated susceptibility to MS. In addition, this MS-associated rs733254 SNP may predict MS onset in females from the Arabian Gulf population.
\end{abstract}

Correspondence to: Professor M. Dahmani Fathallah, Department of Life Sciences, Biotechnology Program, College of Graduate Studies, Arabian Gulf University, Building 293, Road 2904, Manama 329, Bahrain

E-mail: d.fathallah@agu.edu.bh

Key words: multiple sclerosis, Arabian Gulf population, SNPs, genetic, association, susceptibility, gender

\section{Introduction}

Multiple sclerosis (MS) is a chronic inflammatory disease affecting the central nervous system (CNS) in adults. It leads to myelin loss, varying degrees of axonal pathology, and progressive disability (1). The causes of MS remain largely unknown; however, genetic and environmental components are involved in susceptibility and disease outcome, however their relative roles are poorly understood (2).

No cure currently exists, however, the variety of available treatments is broadening, particularly for the relapsing-remitting form of MS (RRMS) $(3,4)$. The use of different formulations of interferon- $\beta$ (IFN- $\beta$ ) and glatiramer acetate has reduced the relapse rate and the development of new lesions (as detected by magnetic resonance imaging) (5). More therapeutic agents to treat MS, and the development of biomarkers to predict treatment response are required (6). Genome-wide association studies (GWAS) have notably contributed to the understanding of MS susceptibility. The final aim of GWAS is to use genetic risk factors to make predictions regarding susceptibility to the disease, identify the underlying molecular mechanism and, thus, facilitate the development of novel prevention and therapeutic strategies.

A total of 23 non-human leukocyte antigen (HLA) loci associated with MS were identified by 7 independent GWAS and 1 meta-analysis conducted between 2007 and 2011 (7-11). 10 GWAS have been performed in MS subjects. There were follow-up studies that resulted in the identification of 16 confirmed loci with genome-wide significance. Numerous risk variants are located at or near genes with central immunological functions. The majority are associated with other autoimmune diseases (12). The Wellcome Trust Case Control Consortium 2 in collaboration with the International MS Genetics Consortium has recently completed the largest MS GWAS to date and increased the number of non-HLA genetic loci associated with MS to 57 loci (13). However, additional genetic and functional studies are required to identify the function of these relevant genes and pathways. In 2009, Comabella et al (14) published a whole-genome study investigating single nucleotide polymorphisms (SNPs) associated 
with IFN- $\beta$ therapy response. Top-scoring intragenic SNPs are localized in the following genes: Zinc finger and AT hook domain containing (ZFAT), glutamate receptor, ionotropic, AMPA 3 (GRIA3), StAR-related lipid transfer (START) domain containing 13 (STARD13), adenosine deaminase, RNA-specific (ADAR), zinc finger homeobox 4 (ZFHX4), interferon $(\alpha, \beta$ and $\omega)$ receptor 2 (IFNAR2) and citron Rho-interacting serine/threonine kinase (CIT). The GRIA3 gene encodes an AMPA-type glutamate receptor.

It has been reported that glutamate is important in the CNS (15) and the majority of the excitatory synaptic transmission in the CNS is mediated by AMPA-type glutamate receptors expressed in oligodendrocytes (16). Oligodendrocytes are highly susceptible to excitotoxic signals mediated by AMPA receptors, this indicates that oligodendrocyte excitotoxicity may be implicated in the pathogenesis of demyelinating disorders, including MS (17). CIT is found on postsynaptic sites of glutamatergic and GABAergic neurons interacting with a scaffold protein, postsynaptic density protein 95 (18), predicting potential cross-talk between these two signaling pathways. This is important in numerous aspects of neural plasticity.

In 2005, Leyva et al (19) proposed a role for the IFNAR pathway in susceptibility to MS. ADAR is an IFN-inducible RNA-specific adenosine deaminase implicated in the editing of viral RNA transcripts and cellular pre-mRNAs. Liu and Samuel (20) associated increases in ADAR levels to glutamate receptor function. ZFAT and ZFHX4 genes encode for zinc finger proteins, while STARD13 encodes for a GTPase-activating enzyme. In addition, their possible association with response to IFN- $\beta$ or MS susceptibility remains unknown.

However, none of these genes are currently confirmed as MS risk genes. Genetic associations with MS are less studied in individuals from the Arabian Peninsula. To date, Al Jumah et al (21) has reported the only genetic study confirming a subset of SNPs identified by GWAS as MS-associated in Saudi MS patients. This suggests that further studies of MS genetic susceptibility in the Arabian Peninsula population are required.

The Arab Gulf population has a lower risk of MS compared to Europeans and white Americans. However, recent studies demonstrated an increase in incidence and prevalence of MS, particularly among women within the Arab Gulf region (31-55 MS cases $/ 100,000$ individuals) $(22,23)$. Considering the geographic nature of environmental and genetic factors contributing to the development of MS, the present study investigated the potential role of polymorphisms in the genes encoding ZFAT, GRIA3, STARD13, ADAR, ZFHX4, IFNAR2 and CIT on MS susceptibility.

\section{Materials and methods}

Sample collection. A group of unrelated Arab patients with RRMS were recruited for the current study from different centers in the Arabian Gulf [156 subjects ( 88 women and 68 men)]. MS patients were clinically diagnosed with MS according to McDonald's criteria (24). Disease severity was measured using the Expanded Disability Status Score (EDSS) (25); 96 out of 156 MS patients were treated with IFN- $\beta$. However, the remaining 60 patients received other treatments, including Gilenya, Tysabri (natalizumab), carbamazepine or steroids (cortisone). Patients were considered responders to IFN- $\beta$ when there were no relapses with any increase in the EDSS score during the follow-up period (2 years). Non-responders were those who experienced $\geq 1$ relapses with an increment of at least one point in the EDSS score during the two years of treatment (26). A total of 68 patients were considered responders and 17 as non-responders to IFN- $\beta$ treatment, in addition $11 \mathrm{MS}$ patients did not have complete records for EDSS and relapse scores. The control group included 112 healthy individuals, 64 women and 48 men, from the same region with no personal or familial (first-degree relatives) history of autoimmune diseases. All participants provided written informed consent and the present study was approved by the ethics committee of the Arabian Gulf University (Manama, Bahrain).

SNP selection and genotyping. Seven SNPs were previously reported to be associated with response to INF- $\beta$ treatment: rs12557782 in GRIA3, rs7308076 in CIT, rs2229857 in ADAR, rs733254 in ZFAT, rs9527281 in STARD13, rs11787532 in ZFHX4, and rs2248202 in IFNAR2) (14). Genomic DNA was isolated from blood samples using the Blood \& Cell Culture DNA Mini kit, and EZ1 DNA Investigator Kit (Qiagen, Inc., Valencia, CA, USA) according to the manufacturer's protocols. SNP genotyping was performed by polymerase chain reaction (PCR)-restriction fragment length polymorphism (RFLP) and KBioscience Competitive Allele-Specific PCR genotyping technique (KASP) methods. The present study genotypes three SNP, rs7308076 CIT, rs11787532 ZFHX4, and rs2248202 IFNAR2 with only the KASP method due to the lack of specific restriction sites. Primer sequences used for RFLP were designed based on the GenBank reference sequence for each gene, as follows: Forward, 5'-TCACACATTGGTATG ATTATTTGATTC-3' and reverse, 5'-TTTGATCCTACTTGA ATGTAACTGC-3' for rs12557782 GRIA3 (NM_000828.4), restriction enzyme HeaIII; forward, 5'-AAAGGCAGGGGG ATGTCTAT-3' and reverse, 5'-ATGATGGCTCGAAAC TCACC-3' for rs2229857 ADAR (NM_0,010,25107.2), restriction enzyme MboII; forward, 5'-AGTGACGCTGCCTCT TCTTC-3' and reverse, 5'-CCCTGAGTACCCTTTGGTCA-3' for rs733254 ZFAT (NM_02,0863.3), restriction enzyme $\mathrm{XbaI}$; and: forward, 5'-GGTAGGAGGATTTCGAAGCA-3' and reverse, 5'-GGTGGCTAAGGAAACCTTGA-3' for rs9527281 STARD13 (NM_0,012,43476.2), restriction enzyme Hyp 188III. The RFLP was conducted using the GoTaq Flexi DNA polymerase (Promega Corporation, Madison, WI, USA), in a DNA Engine Tetrad 2 (Bio-Rad Laboratories, Inc., Hercules, CA, USA) thermal cycler with conditions as follows: Initial denaturation at $95^{\circ} \mathrm{C}$ for $5 \mathrm{~min} ; 34$ cycles of $95^{\circ} \mathrm{C}$ for $1 \mathrm{~min}, 60.5^{\circ} \mathrm{C}$ for $45 \mathrm{sec}$, and $72^{\circ} \mathrm{C}$ for $1 \mathrm{~min}$; followed by a final extension at $72^{\circ} \mathrm{C}$ for $7 \mathrm{~min}$.

A total of seven SNPs were genotyped using KASP version 4.0 at Genoscreen (Lille, France). Briefly, KASP genotyping system allows SNPs to be detected via fluorescence resonance energy transfer (FRET). For each SNP, one common reverse primer and two allele-specific forward primers (that differ at their $3^{\prime}$ ends) were designed by Genoscreen. The DNA samples were transferred into 96-well plates and genotyped according to the KASP PCR protocol. Genotyping reactions were performed in 
Table I. Demographic and clinical characteristics of subjects included in the present study.

\begin{tabular}{|c|c|c|c|}
\hline Parameter & Control $(n=112)$ & MS patients $(n=156)$ & P-value \\
\hline \multicolumn{4}{|l|}{ Gender } \\
\hline Female & $64(42.1 \%)$ & $88(57.9 \%)$ & 0.905 \\
\hline Male & $48(41.4 \%)$ & $68(58.6 \%)$ & 0.905 \\
\hline \multicolumn{4}{|l|}{ Age, years } \\
\hline Female & $29.59 \pm 1.086$ & $33.63 \pm 1.021$ & 0.392 \\
\hline Male & $28.46 \pm 1.266$ & $33.56 \pm 1.006)$ & 0.188 \\
\hline \multicolumn{4}{|c|}{ Age at disease onset, years } \\
\hline Female & & $27.33 \pm 0.892$ & 0.607 \\
\hline Male & & $26.03 \pm 0.995$ & 0.607 \\
\hline \multicolumn{4}{|l|}{ EDSS score } \\
\hline Female & & $2.7 \pm 0.179$ & \\
\hline Male & & $2.9 \pm 0.256$ & \\
\hline
\end{tabular}

Results are presented as the mean \pm standard deviation. MS, multiple sclerosis; EDSS, expanded disability status scale.

Table II. Summary of selected single nucleotide polymorphisms.

\begin{tabular}{llcl}
\hline dbSNP ID & Gene & Location & Position \\
\hline rs733254 & ZFAT & 8 & Intron 3 \\
rs12557782 & GRIA3 & X & Intron 2 \\
rs2229857 & ADAR & 1 & Exon 2/missense \\
rs9527281 & STARD13 & 13 & Intron 1 \\
rs11787532 & ZFHX4 & 8 & Intron 3 \\
rs7308076 & CIT & 12 & Intron 9 \\
rs2248202 & IFNAR2 & 21 & Intron 1 \\
\hline
\end{tabular}

ZFAT, zinc finger and AT hook domain containing; GRIA3, glutamate receptor, ionotropic, AMPA 3; ADAR, adenosine deaminase, RNA-specific; STARD13, StAR-related lipid transfer (START) domain containing 13; ZFHX4, zinc finger homeobox 4; CIT, citron Rho-interacting serine/threonine kinase; IFNAR2, interferon $(\alpha, \beta$ and $\omega$ ) receptor 2 .

HC16 thermal cyclers (LGC, Ltd., Teddington, UK) in a final volume of $5 \mu \mathrm{l}$ containing $2.5 \mu \mathrm{l}$ genomic DNA (12 ng/ $\mu \mathrm{l}), 2.5 \mu \mathrm{l}$ of $2 \mathrm{X}$ KASP reaction mix (containing Taq polymerase enzyme and ROX passive reference dye; KBioscience; LGC, Ltd.) and $0.07 \mu 1$ of the KASP assay primer mix (KBioscience; LGC, Ltd.). The following cycling conditions were used: A hot-start Taq polymerase activation step at $94^{\circ} \mathrm{C}$ for $15 \mathrm{~min}$; followed by 10 touchdown cycles of $94^{\circ} \mathrm{C}$ for $20 \mathrm{sec}, 65-57^{\circ} \mathrm{C}$ for $1 \mathrm{~min}$ (decreasing $0.8^{\circ} \mathrm{C} /$ cycle); and 26 cycles of $94^{\circ} \mathrm{C}$ for $20 \mathrm{sec}$ and $57^{\circ} \mathrm{C}$ for $1 \mathrm{~min}$. Fluorescence detection of the reactions was performed using a FRET reading instrument, and PHERAstar plate reader (BMG Labtech $\mathrm{GmbH}$, Ortenberg, Germany) and genotyping data was analyzed with KlusterCaller software (Kbioscience; LGC, Ltd.).

Statistical analysis. Statistical analysis was conducted using SPSS 19 (IBM SPSS, Armonk, NY, USA). Independent samples t-test and $\chi^{2}$ test were applied to compare the basic demographic data between case and control groups. The Hardy-Weinberg exact equilibrium test and the analysis of SNP association with MS (based on logistic regression) were performed using the web-tool SNPStats (bioinfo.iconcologia.net/SNPstats, provided by the Catalan Institute of Oncology, Spain) (27) and two-way contingency table analysis (statpages.org/ctab2x2.html). The SHEsis software platform (http://analysis.bio-X.cn/SHEsisMain.htm) was also used for the association study $(28,29)$. CaTS software (http://csg.sph.umich.edu/abecasis/CaTS/) performed power calculations for the genetic association study. The Akaike Information Criterion (AIC) selected the genetic model illustrating the association of SNPs with MS. P<0.05 was considered to indicate a statistically significant difference.

\section{Results}

A total of 268 unrelated individuals with 112 controls, 64 female and 48 male) and 156 MS cases ( 88 female and 68 male) were observed in the present study. Table I provides a summary of the demographic and clinical features of the study participants. No notable differences were detected between the MS and control groups.

Table II presents the 7 SNPs described by Comabella et al (14) as significantly associated with INF- $\beta$ therapy in Spanish MS patients. The mean SNPs call rate was $98 \%$ in patients and controls. All the tested SNPs followed the Hardy-Weinberg equilibrium, with the exception of rs12557782 in the GRIA3 gene. Thus, this polymorphism was discarded from further analyses.

Tables III and IV indicate the association of each SNP with MS risk in the 156 patients (for all patients, and women and men separately). Of the six SNPs, ZFAT rs733254 SNPs exhibited an allelic association with MS and $\mathrm{P}<0.01$ (Table III). The $\mathrm{T}$ allele frequency is significantly higher in the control group compared with the MS patients group [odds ratio $(\mathrm{OR})=0.53$ and 95\% confidence interval (CI), 0.37-0.77); $\mathrm{P}=0.0004)$. 


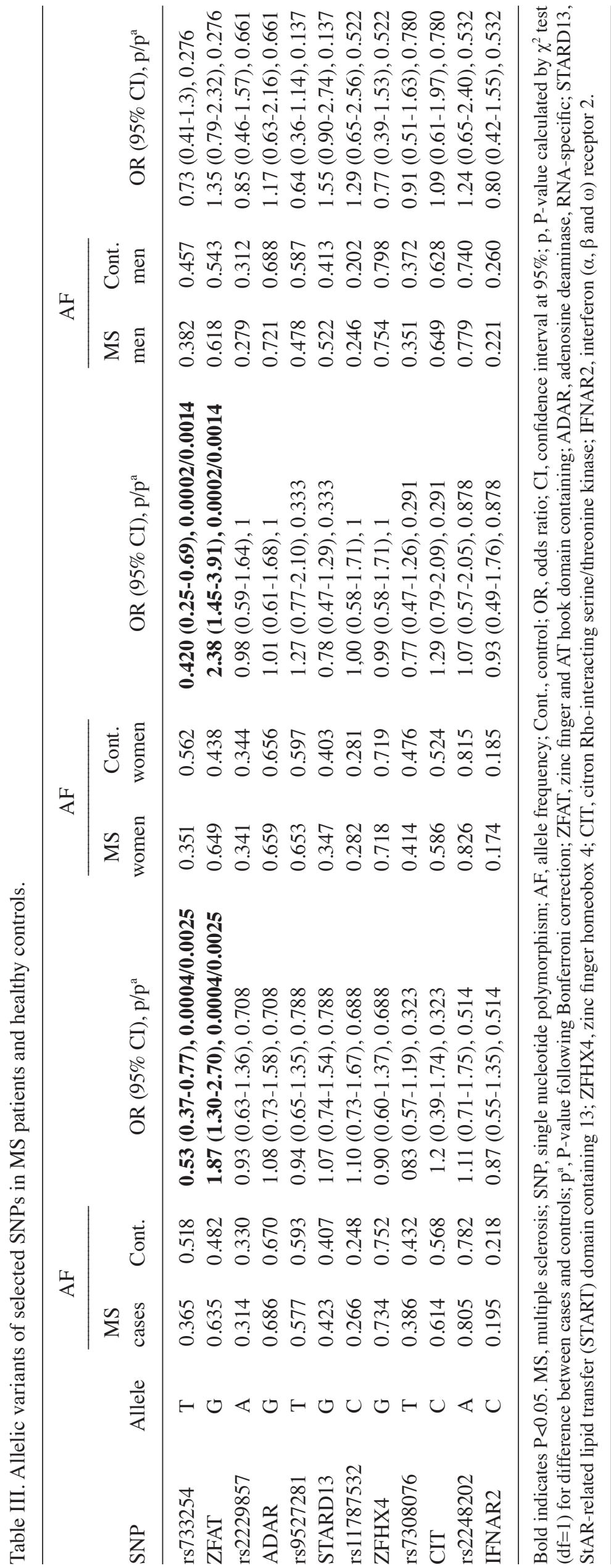




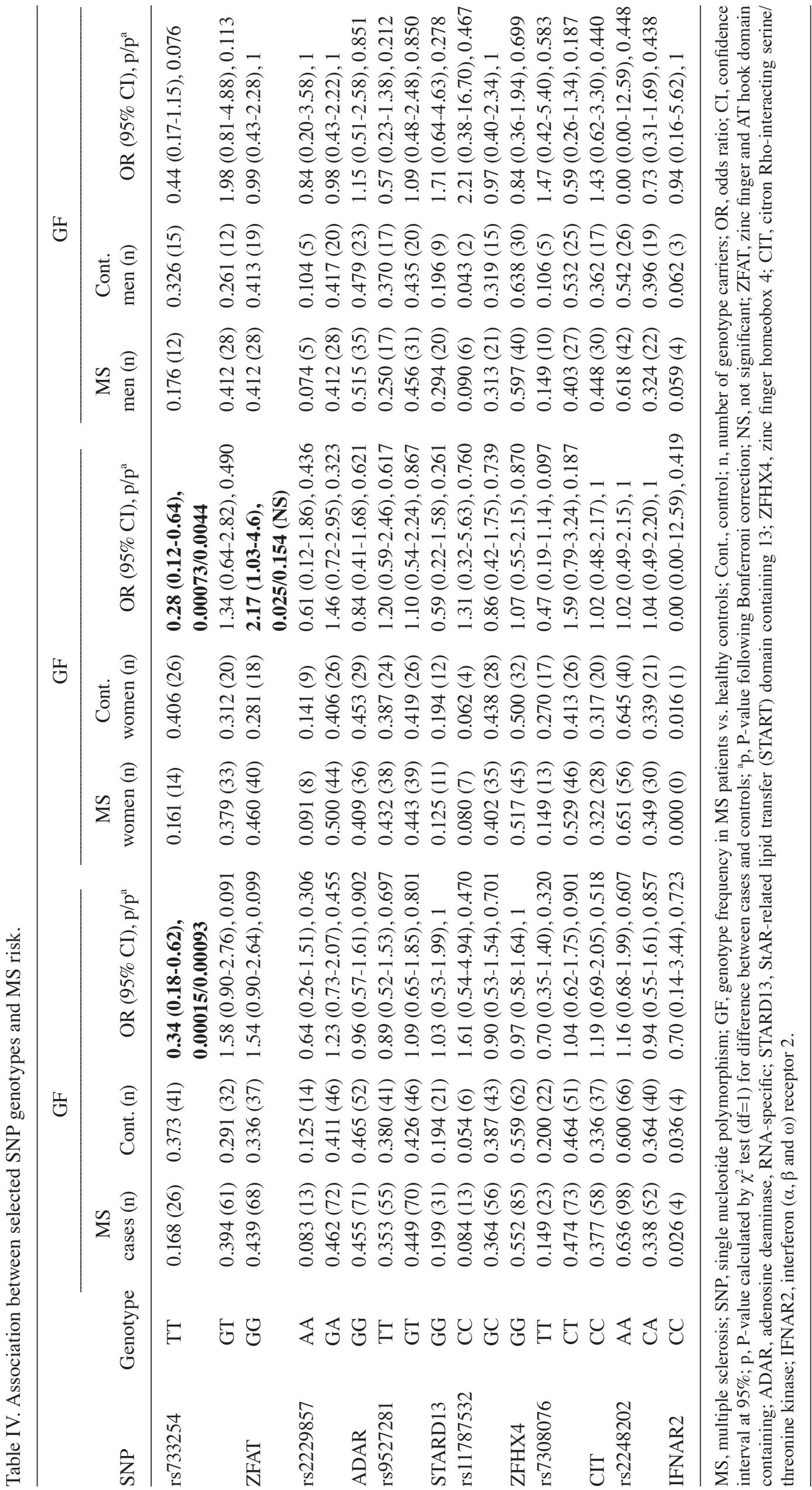


Table V. Association between rs733254 ZFAT and multiple sclerosis risk based on logistic regression test.

\begin{tabular}{lcccr}
\hline SNP & Model & OR $(95 \%$ CI $)$ & P-value & AIC \\
\hline rs733254 & C (GT vs. GG) & $0.98(0.53-1.80)$ & 0.0003 & 336.5 \\
& C (TT vs. GG) & $0.30(0.15-0.58)$ & 0.0003 & 346.7 \\
& D [(GT+TT) vs. GG] & $0.59(0.35-1.01)$ & 0.052 & $\mathbf{3 3 4 . 5}$ \\
ZFAT & R [TT vs. (GG+GT)] & $\mathbf{0 . 3 0}(\mathbf{0 . 1 7 - 0 . 5 5})$ & $\mathbf{0 . 0 0 0 1}$ & 338.8 \\
\hline
\end{tabular}

SNP, single nucleotide polymorphism; ZFAT, zinc finger and AT hook domain containing; C, codominant model; D, dominant model; R, recessive model; L-A, Log-additive model; OR, odds ratio; CI, confidence interval at 95\%; AIC, akaike information citerion. The best model is indicated in bold (the lowest AIC value).

However, a notable over-representation of the $\mathrm{G}$ allele $[\mathrm{OR}=1.87$ (95\% CI, 1.30-2.70); $\mathrm{P}=0.0004]$ was observed among MS cases (Table III). The aforementioned associations are more significant in women than in the total group $[\mathrm{P}=0.0004, \mathrm{OR}=0.420$ (95\% CI, 0.25-0.69); $\mathrm{P}=0.0002, \mathrm{OR}=2.38$ (95\% CI, 1.45-3.91), respectively; Table III].

Following application of the highly conservative Bonferroni correction for multiple tests, the two allelic associations remained significantly associated with MS susceptibility (Table III). At $\alpha=0.05$, with the sample size used in the present study, the power for the significant association was $92 \%$. These associations were not observed in men (Table III). The same was observed for TT and GG genotype frequencies of ZFAT rs733254 (Table IV). In female MS patients, the variant $\mathrm{GG}$ was significantly associated with increased risk of MS [OR=2.17 (95\% CI, 1.03-4.6); $\mathrm{P}=0.025]$. Subsequent to Bonferroni correction for multiple testing, the statistical significance was lost $[\mathrm{OR}=2.17$ (95\% CI, 1.03-4.6); $\mathrm{P}=0.154]$. However, the genotype TT of ZFAT rs733254 was significantly associated with a decreased risk of MS $[\mathrm{OR}=0.28$ (95\% CI, 0.12-0.64), $\mathrm{P}=0.00073$ ], and remained statistically significant following Bonferroni correction $[\mathrm{OR}=0.28(95 \%$ CI, 0.12-0.64); $\mathrm{P}=0.0044]$. At the same time, these associations were not observed in men.

None of the selected SNPs in ADAR, STARD13, ZFHX4, $C I T$ and IFNRA2 genes demonstrated any significant association with MS risk, even when analyzing gender separately (Tables III and IV).

Another model of association analysis was performed by logistic regression test. Consistent with the AIC, the ZFAT rs733254 was observed to be associated with MS by a recessive model (Table V). As demonstrated by the OR, the variant TT was associated with reduced susceptibility to $\mathrm{MS}(\mathrm{OR}=0.30$ (95\% CI, 0.17-0.55); $\mathrm{P}=0.0001]$.

The findings of the present study indicate that $Z F A T$ rs733254 may be critical to MS development in women, however, this requires validation in studies with a larger sample size. However, in the male cohort it exhibited no significant association with MS.

\section{Discussion}

GWAS are conducted in a defined ethnic setting in order to identify genes involved in the onset of numerous diseases.
Nevertheless, validation of these data by independent studies in different ethnic groups remains an important area of research. Recent successes using GWAS approaches have identified seven gene variants that contribute to the IFN- $\beta$ therapy response in Spanish MS patients (14). However, none of these genes were previously confirmed as a MS risk gene. To investigate these findings in a different ethnic setting and determine population variability in the genome of MS patients, a genetic study of the reported seven SNPs in the Arabian Gulf population was conducted.

The present case-control study determined that rs733254 $\mathrm{T}>\mathrm{G}$ in intron 3 of the ZFAT gene was significantly associated with a risk of MS. To the best of our knowledge, no previous studies have demonstrated such an association. The rs733254 $\mathrm{T}$ allele was a statistically significant protective factor against MS [OR=0.53 (95\% CI, 0.37-0.77); $\mathrm{P}=0.0025]$. Furthermore, it was observed that the recessive model is the most fitting genetic model for ZFAT rs733254 (Table V). As indicated by the OR, the variant TT was associated with reduced susceptibility to MS [OR=0.30 (95\% CI, 0.17-0.55); $\mathrm{P}=0.0001]$. Furthermore, the $\mathrm{G}$ allele was significantly associated with increased risk of MS [OR=1.87 (95\% CI, 1.30-2.70); $\mathrm{P}=0.0025]$.

The observed risk with the $\mathrm{G}$ allele of ZFAT SNP is the same as in the previous Comabella et al (2009) GWAS study (14) indicating that ZFAT rs733254 $\mathrm{G}$ allele may be critical to MS development and is a contributing factor to INF- $\beta$ therapy response in MS patients. However, the sample size of INF- $\beta$ non-responder MS patients (68 patients were recorded as responders, 17 as non-responders to IFN- $\beta$ treatment) in the present study was not sufficiently large to replicate the GWAS data mentioned above. In the present study, the GG genotype of ZFAT SNP was identified as the risk genotype in the female group with frequencies of 0.460 in patients and 0.281 in controls. In the Spanish population, GG + GT genotypes were reported as risk genotypes associated with INF- $\beta$ therapy response in MS.

No significant associations of rs7308076 CIT, rs2229857 $A D A R$, rs9527281 STARD13, rs11787532 ZFHX4, or rs2248202 IFNAR2 with MS in the Arabian Gulf population were observed in the present study. Associations of certain SNPs are more consistent among populations than other SNPs. This may depend on allelic frequencies, localization in genes and chromosomes, different linkage disequilibrium patterns in populations, and the different evolutionary history of genes that affect complex diseases (30). 
The present study demonstrates, to the best of our knowledge, for the first time, that rs733254 $\mathrm{T}>\mathrm{G}$ variants in ZFAT gene confers risk for MS in the Arabian Gulf population in a gender-specific manner. ZFAT was highly associated with MS in women, but less so when the analysis was performed in men and women. ZFAT rs733254 may be key in MS development in women and could be validated in studies with a larger sample size. It was hypothesized that the gender-specific effects of a particular loci on MS susceptibility may result from gender-dependent differences in immune response (31). Until recently, genes of the immune system have been observed to be associated with MS in a gender-specific way. SNPs in chemokine (C-X-C motif) ligand 16 (32), brain-derived neurotrophic factor (33) and HLA-DRB1*1501 (34) are associated with MS exclusively or with high significance in women.

The ZFAT gene was initially identified as a candidate gene for autoimmune thyroid disease via linkage and association analyses using 515 Japanese patients and 526 controls $(35,36)$. The ZFAT gene encodes a 1243-amino acid residue protein, including one AT-hook and 18 C2H2 zinc finger domains (37). The mouse ZFAT protein is expressed in $\mathrm{B}$ and $\mathrm{T}$ lymphocytes. ZFAT is a critical transcription factor regulating the genes involved in immune responses (37). Furthermore, the ZFAT protein is a key regulator involved in apoptosis and cell survival for immune-associated cells (38).

Genetic analyzes conducted in the last decade have demonstrated a novel profile of MS pathogenesis and indicated a high degree of variability between individuals and populations. To establish efficient treatments for MS based on the pathogenesis and prognosis of individuals and to pave the way for novel MS therapies tailored to patients' genetic background, further studies are required in different ethnic settings. Future large-scale GWAS (including other populations worldwide) and the meta-analysis of these studies may describe the complete profile of MS.

In conclusion, the present study provides supporting evidence for gender differences in the association of the ZFAT gene (rs733254 $\mathrm{T}>\mathrm{G}$ ) with the pathogenesis of MS in the Arabian Gulf population. The current study suggests a genetic basis may account for the reported gender differences in MS risk, which demonstrates that genetic polymorphisms within immune-associated genes may have different effects in women and men. This suggests a novel research area in the genetics of the immune system response to autoimmune stimuli.

\section{Acknowledgements}

The authors wish to acknowledge Dr. Isa Alsharoqi (Department of Clinical Neurosciences, Salmaniya Medical Complex, Manama, Bahrain) and Dr. Farzana Al Sayed (Gulf Diabetes Specialist Center, Manama, Bahrain) for facilitating access to patients. The present study was supported by a research grant from the Arabian Gulf University (grant no. BIO01).

\section{References}

1. Noseworthy JH, Lucchinetti C, Rodriguez $M$ and Weinshenker BG: Multiple sclerosis. N Engl J Med 343: 938-952, 2000.

2. Dyment DA, Ebers GC and Sadovnick AD: Genetics of multiple sclerosis. Lancet Neurol 3: 104-110, 2004.
3. Bakshi R: The new era of multiple sclerosis therapeutics. Neurotherapeutics 10: 1, 2013.

4. Castro-Borrero W, Graves D, Frohman TC,Flores AB,Hardeman P, Logan D, Orchard M, Greenberg B and Frohman EM: Current and emerging therapies in multiple sclerosis: A systematic review. Ther Adv Neurol Disord 5: 205-220, 2012.

5. McGraw CA and Lublin FD: Interferon beta and glatiramer acetate therapy. Neurotherapeutics 10: 2-18, 2013.

6. Happe LE: Choosing the best treatment for multiple sclerosis: Comparative effectiveness, safety and other factors involved in disease-modifying therapy choice. Am J Manag Care 19 (Suppl 17): S332-S342, 2013.

7. Baranzini SE, Wang J, Gibson RA, Galwey N, Naegelin Y Barkhof F, Radue EW, Lindberg RL, Uitdehaag BM, Johnson MR, et al: Genome-wide association analysis of susceptibility and clinical phenotype in multiple sclerosis. Hum Mol Genet 18: 767-778, 2009.

8. Comabella M, Craig DW, Camiña-Tato M, Morcillo C, Lopez C, Navarro A, Rio J, BiomarkerMS Study Group, Montalban X and Martin R: Identification of a novel risk locus for multiple sclerosis at $13 \mathrm{q} 31.3$ by a pooled genome-wide scan of 500,000 single nucleotide polymorphisms. PLoS One 3: e3490, 2008.

9. International Multiple Sclerosis Genetics Consortium, Hafler DA, Compston A, Sawcer S, Lander ES, Daly MJ, De Jager PL, de Bakker PI, Gabriel SB, Mirel DB, et al: Risk alleles for multiple sclerosis identified by a genomewide study. N Engl J Med 357: 851-862, 2007.

10. JakkulaE,Leppä V,Sulonen AM,VariloT, KallioS, Kemppinen A, Purcell S, Koivisto K, Tienari P, Sumelahti ML, et al: Genome-wide association study in a high-risk isolate for multiple sclerosis reveals associated variants in STAT3 gene. Am J Human Genet 86: 285-291, 2010.

11. Patsopoulos NA; Bayer Pharma MS Genetics Working Group; Steering Committees of Studies Evaluating IFN $\beta-1 b$ and a CCR1-Antagonist; ANZgene Consortium; GeneMSA; International Multiple Sclerosis Genetics Consortium, Esposito F, Reischl J, Lehr S, Bauer D, et al: Genome-wide meta-analysis identifies novel multiple sclerosis susceptibility loci. Annl Neurol 70: 897-912, 2011.

12. Kemppinen A, Sawcer S and Compston A: Genome-wide association studies in multiple sclerosis: Lessons and future prospects. Brief Funct Genomics 10: 61-70, 2011.

13. International Multiple Sclerosis Genetics Consortium; Wellcome Trust Case Control Consortium 2, Sawcer S, Hellenthal G, Pirinen M, Spencer CC, Patsopoulos NA, Moutsianas L, Dilthey A, Su Z, et al: Genetic risk and a primary role for cell-mediated immune mechanisms in multiple sclerosis. Nature 476: 214-219, 2011.

14. Comabella M, Craig DW, Morcillo-Suárez C, Rio J, Navarro A, Fernandez M, Martin R and Montalban X: Genome-wide scan of 500,000 single-nucleotide polymorphisms among responders and nonresponders to interferon beta therapy in multiple sclerosis. Arch Neurol 66: 972-978, 2009.

15. Barnes GN and Slevin JT: Ionotropic glutamate receptor biology: Effect on synaptic connectivity and function in neurological disease. Curr Med Chem 10: 2059-2072, 2003.

16. Mayer ML and Armstrong N: Structure and function of glutamate receptor ion channels. Annu Rev Physiol 66: 161-181, 2004.

17. Werner P, Pitt D and Raine CS: Glutamate excitotoxicity-a mechanism for axonal damage and oligodendrocyte death in multiple sclerosis? J Neural Transm Suppl 2000: 375-385.

18. Furuyashiki T, Fujisawa K, Fujita A, Madaule P, Uchino S, Mishina M, Bito H and Narumiya S: Citron, a Rho-target, interacts with PSD-95/SAP-90 at glutamatergic synapses in the thalamus. J Neurosci 19: 109-118, 1999.

19. Leyva L, Fernández O, Fedetz M, Blanco E, Fernández VE, Oliver B, León A, Pinto-Medel MJ, Mayorga C, Guerrero M, et al: IFNAR1 and IFNAR2 polymorphisms confer susceptibility to multiple sclerosis but not to interferon-beta treatment response. J Neuroimmunol 163: 165-171, 2005.

20. Liu Y and Samuel CE: Editing of glutamate receptor subunit B pre-mRNA by splice-site variants of interferon-inducible double-stranded RNA-specific adenosine deaminase ADAR1. J Biol Chem 274: 5070-5077, 1999.

21. Al Jumah M, Al Balwi M, Hussein M, Kojan S, Al Khathaami A, Al Fawaz M, Al Muzaini B, Jawhary A and Al Abdulkareem I: Association of SNPs rs 6498169 and rs10984447 with multiple sclerosis in Saudi patients: A model of the usefulness of familial aggregates in identifying genetic linkage in a multifactorial disease. Mult Scler 18: 1395-1400, 2012. 
22. Bohlega S, Inshasi J, Al Tahan AR, Madani AB, Qahtani H and Rieckmann P: Multiple sclerosis in the Arabian Gulf countries: A consensus statement. J Neurol 260: 2959-2963, 2013.

23. Deleu D, Mir D, Al Tabouki A, Mesraoua R, Mesraoua B, Akhtar N, Al Hail H, D'souza A, Melikyan G, Imam YZ, et al: Prevalence, demographics and clinical characteristics of multiple sclerosis in Qatar. Mult Scler 19: 816-819, 2013.

24. Polman CH, Reingold SC, Banwell B, Clanet M, Cohen JA, Filippi M, Fujihara K, Havrdova E, Hutchinson M, Kappos L, et al: Diagnostic criteria for multiple sclerosis: 2010 evisions to the McDonald criteria. Ann Neurol 69: 292-302, 2011.

25. Kurtzke JF: Rating neurologic impairment in multiple sclerosis: An expanded disability status scale (EDSS). Neurology 33 1444-1452, 1983.

26. Rio J, Nos C, Tintoré M, Téllez N, Galán I, Pelayo R, Comabella M and Montalban X: Defining the response to interferon-beta in relapsing-remitting multiple sclerosis patients. Ann Neurol 59: 344-352, 2006

27. Solé X, Guinó E, Valls J, Iniesta R and Moreno V: SNPStats: A web tool for the analysis of association studies. Bioinformatics 22 1928-1929, 2006.

28. Shi YY and He L: SHEsis, a powerful software platform for analyses of linkage disequilibrium, haplotype construction, and genetic association at polymorphism loci. Cell Res 15: 97-98, 2005.

29. Li Z, Zhang Z, He Z, Tang W, Li T, Zeng Z, He L and Shi Y: A partition-ligation-combination-subdivision EM algorithm for haplotype inference with multiallelic markers: Update of the SHEsis (http://analysis.bio-x.cn). Cell Res 19: 519-523, 2009.

30. Marigorta UM, Lao O, Casals F, Calafell F, Morcillo-Suárez C, Faria R, Bosch E, Serra F, Bertranpetit J, Dopazo H, et al: Recent human evolution has shaped geographical differences in susceptibility to disease. BMC Genomics 12: 55, 2011.
31. Khalid R: Contributing factors in multiple sclerosis and the female sex bias. Immunol Lett 162: 223-232, 2014

32. Stojković L, Stanković A, Djurić T, Dinčić E, Alavantić D and Zivković M: The gender-specific association of CXCL16 A181V gene polymorphism with susceptibility to multiple sclerosis and its effects on PBMC mRNA, and plasma soluble CXCL16 levels: Preliminary findings. J Neurol 261: 1544-1551, 2014.

33. Mirowska-Guzel D, Mach A, Gromadzka G, Czlonkowski A and Czlonkowska A: BDNF A196G and C270T gene polymorphisms and susceptibility to multiple sclerosis in the Polish population. Gender differences. J Neuroimmunol 193: 170-172, 2008.

34. Benešová $Y$, Vašků $A$, Stourač $P$, Hladíková $M$, Fiala $A$ and Bednařík J: Association of HLA-DRB1*1501 tagging rs3135388 gene polymorphism with multiple sclerosis. J Neuroimmunol 255: 92-96, 2013.

35. Shirasawa S, Harada H, Furugaki K, Akamizu T, Ishikawa N, Ito K, Ito K, Tamai H, Kuma K, Kubota S, et al: SNPs in the promoter of a B cell-specific antisense transcript, SAS-ZFAT, determine susceptibility to autoimmune thyroid disease. Hum Mol Genet 13: 2221-2231, 2004.

36. Sakai K, Shirasawa S, Ishikawa N, Ito K, Tamai H, Kuma K, Akamizu T, Tanimura M, Furugaki K, Yamamoto K, et al: Identification of susceptibility loci for autoimmune thyroid disease to 5q31-q33 and Hashimoto's thyroiditis to 8q23-q24 by multipoint affected sib-pair linkage analysis in Japanese. Hum Mol Genet 10: 1379-1386, 2001.

37. Koyanagi M, Nakabayashi K, Fujimoto T, Gu N, Baba I, Takashima Y, Doi K, Harada H, Kato N, Sasazuki T and Shirasawa S: ZFAT expression in B and T lymphocytes and identification of ZFAT-regulated genes. Genomics 91: 451-457, 2008.

38. Fujimoto T, Doi K, Koyanagi M, Tsunoda T, Takashima Y, Yoshida Y, Sasazuki T and Shirasawa S: ZFAT is an antiapoptotic molecule and critical for cell survival in MOLT-4 cells. FEBS Lett 583: 568-572, 2009. 